\title{
Angiotensin-Converting Enzyme Inhibitors and Angiotensin Receptor Blockers after Acute Kidney Injury: Friend, Foe, or Acquaintance?
}

\author{
Steven G. Coca \\ Division of Nephrology, Department of Medicine, Icahn School of Medicine at Mount Sinai, New York, NY, USA
}

Nephrologists do not have it easy. We frequently deal with "double-edged swords" in our clinical practice. We have drugs that are often good for the kidneys in the long term but can also worsen kidney function during times of stress. In the transplant world, the calcineurin inhibitors are the mainstays of immunosuppression to avoid rejection of the transplanted kidney, but these agents can also contribute to long-term allograft fibrosis. In the nontransplant setting, both angiotensin-converting enzyme inhibitors or angiotensin receptor blockers (an ACEi or ARBs) and sodium-glucose co-transporter 2 inhibitors are nephroprotective in the long term. However, through their effects on periglomerular hemodynamics, systemic blood pressure, and natriuresis, in acute settings and times of stress (i.e., hospitalizations), the one-time "hero's" and protectors of the kidneys are often seen as villains in this context. They are sometimes even labeled as "nephrotoxins" [1]. Regardless of whether or not they receive the "bad actor" label descriptor verbally or in the medical record, they are most often discontinued by treating clinicians during acute kidney injury (AKI). Are the clinicians that stop an ACEi or ARB during or after AKI doing the right thing?

First, there are no randomized controlled trials (RCT) on an ACEi or ARB continuation versus discontinuation in patient's hospitalization with some degree of AKI. Second, everybody, patients and providers alike, prefer to see better "numbers" and especially lower serum creatinine

\section{KARGER}

๑ 2020 S. Karger AG, Basel concentrations. If one is ill, stressed, and has lower blood pressure than usual, the general tendency of continuation of an ACEi or ARB administration will be to worsen glomerular filtration rate (GFR) due to their effects on systemic blood pressure and efferent arteriolar tone. Third, the Kidney Disease Improving Global Outcomes clinical practice guidelines for AKI recommend discontinuation of the agents during AKI. Fourth, what is the downside of withholding an ACEi or ARB for a few days during acute illness, if their protection comes from long-term therapy with these drugs? Lack of an ACEi or ARB treatment over a short time frame should not have an important detrimental impact upon long-term clinical outcomes.

The counterarguments to these viewpoints have been largely intellectual or, some may say, hard-headed, for trying to make the case that an ACEi or ARB should be continued during AKI. Some of the ivory-tower pundits' arguments to continue an ACEi or ARBs are the following: (1) The drop in estimated GFR (eGFR) does not connote true kidney tubular injury. Blood flow to the peritubular capillaries should not necessarily be impeded (unless systemic blood pressure drops low enough) just from the effect on the efferent arteriole [2]. (2) If these meds are stopped, they may or may not be restarted at discharge or as an outpatient. Thus, the properties of an ACEi or $\mathrm{ARB}$ that enable cardiorenal protection were flummoxed by their evil alter-ego that induced discontinuation because of AKI. This conflict begets a vicious cycle. 
Chronic kidney disease (CKD) is the strongest risk factor for AKI. ACEi or ARBs are protective for most forms of CKD, particularly those with proteinuria [3]. These patients who are prone to AKI get the ACEi or ARB stopped and are now stripped of their long-term protection, despite the fact that AKI is often going to coexist in patients with CKD. (3) Given all the data and literature on "AKI to CKD" transition $[4,5]$, it would be expected that an ACEi or ARB, through their vasculoprotective effects and attenuation of tubulointerstitial fibrosis, would actually be the agents of choice to prevent AKI to transition to sustained CKD. To be clear, there are few data to support these arguments, but in the absence of objective data, these are where the 2 camps lie.

Recent studies indicate that post-AKI use of an ACEi or ARB may increase [6] or decrease [7] the risk for recurrent AKI. Even in the study that demonstrated an increased risk for recurrent AKI, the risk for death was actually $15 \%$ lower in those that started an ACEi or ARB post-AKI [6]. While these data are intriguing, these studies were retrospective, and even though propensitymatching techniques were employed, there is no equivalent substitute for randomization.

In this issue of the journal, Hines et al. [8] provide us with results of a multicenter prospective cohort study of 345 hospitalized patients complicated by AKI who attended specialized AKI follow-up clinics between 2013 and 2018. The primary outcome was acute kidney disease (AKD) defined as a serum creatinine $\geq 1.5$ times above pre-AKI baseline at the first clinic visit. At the first outpatient visit, 112 (32\%) had persistent AKD. Among the 163 (47\%) patients who were prescribed an ACEi or an ARB before hospitalization, only $23 \%$ were discharged on one of these agents. The authors found no difference in the rate of AKD in patients discharged on an ACEi or $\mathrm{ARB}$ versus those, not on these agents ( 12.5 vs. $15.0 \%, p=$ $0.530)$. Of the $22(19.6 \%)$ patients with AKD who had acute ACEI or ARB exposure during the hospitalization, there was not associated AKD at the time of first clinic visit (median, interquartile range 52.33, 18-54 days following hospital discharge) [8]. Thus, acute exposure to an ACEI or ARB before or during an episode of AKI was not associated with persistent $\mathrm{AKD}$ at the time of the first clinic visit. This suggests that the receipt of an ACEi or ARB does not impede kidney recovery following AKI. This is in sharp contrast to prevailing recommendations and current practice, based largely on epidemiologic retrospective studies.

The data from previous randomized clinical trials of AKI in other settings support only 2 proven areas where an ACEi or ARB has long-term renal benefits. The first is in the setting of heart failure due to reduced ejection fraction $[8,9]$ and the second is proteinuric kidney disease [3]. In the study by Hines et al. [8], only $24 \%$ had a history of heart failure, and proteinuria was not captured as a characteristic of the cohort. Moreover, only $33 \%$ had CKD at baseline. Thus, we need more data on the role of an ACEi or ARB during and after AKI. However, the study by Hines et al. [8] digs deeper into the $\mathrm{ACEi}$ or ARB use in the AKI conundrum. The remarkable finding of this study is that only 70/163 (43\%) who received an $A C E i$ or $A R B$ prior to hospitalization were taking an ACEi or ARB at the time of the first post-AKI clinic visit. Thus, the majority did NOT restart their ACEi or ARB. One of the reasons was the complete lack of guidance for $72 \%$ of patients about how to manage an ACEi or ARB restart as reflected by the discharge summaries. The authors noted while acute tubular necrosis was associated with a higher risk of AKI, as serum creatinine was higher at discharge, they reported that acute exposure to an ACEi or ARB was not associated with $\mathrm{AKD}$ or inability to recover within $50 \%$ of baseline eGFR. Eureka! This gives us the answer...patients should not have an ACEi or ARB withheld during AKI!

Au contraire mon frère. There are 3 major issues with the findings. First, there is clearly confounding by indication or treatment bias. Those with less severe forms of AKI were more likely to have continued an ACEi or ARB exposure [9]. Thus, it is very hard to disentangle causality. Second, statistical limitations do not provide the possibility to conclude "no association with AKI or inability to recover." The 95\% CI for the association between an ACEi or ARB and AKD was 0.66-2.67 [9]. Thus, a lack of association cannot be determined, as there could be as much as a $34 \%$ protection or greater than a 2.5 -fold increased risk for harm. Third, we do not know what happened to the patients after the post-AKI visit.

In conclusion, while this is noble work, the greatest message from this paper is that most people have an ACEi or ARB withheld post-AKI, and most do not restart an ACEi or ARB after AKI. We really need a RCT that is adequately powered to once and for all provide guidance for this common clinical dilemma. While the issue of an ACEi or ARB exposure in the hospital may be too hot to handle, and the juice may not be worth the squeeze, there is enough equipoise regarding the usage of these agents' posthospitalization for AKI. The nephrology community should embrace an RCT in this setting. 


\section{Acknowledgments}

Dr. Steven G. Coca is a member and has salary support from the CKD Biomarker Consortium (U01DK106962). He is also supported, in part, by the following grants: R01DK115562, R01HL85757, R01DK112258, and U01OH011326.

\section{Disclosure Statement}

Dr. Steven G. Coca is a member of the advisory board of RenalytixAI and owns equity in the same. In the past 3 years, he has received consulting fees from RenalytixAI, Goldfinch Bio, CHF Solutions, Quark Biopharma, Janssen Pharmaceuticals, Takeda Pharmaceuticals, Relypsa, Bayer, and pulse Data.

\section{References}

1 Jones $\mathrm{M}$, Tomson C. Acute kidney injury and 'nephrotoxins': mind your language. Clin Med (Lond). 2018 Oct;18(5):384-6.

2 Perazella MA, Coca SG. Three feasible strategies to minimize kidney injury in 'incipient AKI'. Nat Rev Nephrol. 2013 Aug;9(8):48490.

3 Kent DM, Jafar TH, Hayward RA, Tighiouart $\mathrm{H}$, Landa $\mathrm{M}$, de Jong $\mathrm{P}$, et al. Progression risk, urinary protein excretion, and treatment effects of angiotensin-converting enzyme inhibitors in nondiabetic kidney disease. J Am Soc Nephrol. 2007 Jun;18(6):1959-65.

4 Basile DP, Bonventre JV, Mehta R, Nangaku $\mathrm{M}$, Unwin R, Rosner MH, et al.; ADQI XIII Work Group. Progression after AKI: Under- standing Maladaptive Repair Processes to Predict and Identify Therapeutic Treatments. J Am Soc Nephrol. 2016 Mar;27(3):687-97.

5 Kaushal GP, Shah SV. Challenges and advances in the treatment of AKI. J Am Soc Nephrol. 2014 May;25(5):877-83.

6 Brar S, Ye F, James MT, Hemmelgarn B, Klarenbach S, Pannu N; Interdisciplinary Chronic Disease Collaboration. Association of Angiotensin-Converting Enzyme Inhibitor or Angiotensin Receptor Blocker Use With Outcomes After Acute Kidney Injury. JAMA Intern Med. 2018 Dec;178(12):1681-90.

7 Hsu CY, Liu KD, Yang J, Glidden DV, Tan TC, Pravoverov L, et al. Renin-Angiotensin System Blockade after Acute Kidney Injury
(AKI) and Risk of Recurrent AKI. Clin J Am Soc Nephrol. 2020 Jan;15(1):26-34.

8 Hines A, Li X, Ortiz-Soriano V, Saleh S, Litteral J, Ruiz-Conejo M, et al. Use of Angiotensin-Converting Enzyme Inhibitors/Angiotensin Receptor Blockers and Acute Kidney Disease after an Episode of AKI: A Multicenter Prospective Cohort Study. Am J Nephrol. 2020, DOI: 10.1159/000505893.

9 Flather MD, Yusuf S, Køber L, Pfeffer M, Hall A, Murray G, et al.; ACE-Inhibitor Myocardial Infarction Collaborative Group. Long-term ACE-inhibitor therapy in patients with heart failure or left-ventricular dysfunction: a systematic overview of data from individual patients. Lancet. 2000 May;355(9215):1575-81. 